\title{
Vertex properties of maximum scattered linear sets of $\mathrm{PG}\left(1, q^{n}\right)$
}

\author{
Corrado Zanella and Ferdinando Zullo *
}

\begin{abstract}
In this paper we investigate the geometric properties of the configuration consisting of a subspace $\Gamma$ and a canonical subgeometry $\Sigma$ in $\mathrm{PG}\left(n-1, q^{n}\right)$, with $\Gamma \cap \Sigma=\emptyset$. The idea motivating is that such properties are reflected in the algebraic structure of the linear set which is projection of $\Sigma$ from the vertex $\Gamma$. In particular we deal with the maximum scattered linear sets of the line $\mathrm{PG}\left(1, q^{n}\right)$ found by Lunardon and Polverino in [27] and recently generalized by Sheekey in [37. Our aim is to characterize this family by means of the properties of the vertex of the projection as done by Csajbók and the first author of this paper for linear sets of pseudoregulus type. With reference to such properties, we construct new examples of scattered linear sets in $\mathrm{PG}\left(1, q^{6}\right)$, yielding also to new examples of MRD-codes in $\mathbb{F}_{q}^{6 \times 6}$ with left idealiser isomorphic to $\mathbb{F}_{q^{6}}$.
\end{abstract}

AMS subject classification: 51E20, 05B25, 51E22

Keywords: Linear set, linearized polynomial, $q$-polynomial, finite projective line, subgeometry, scattered linear set

\section{Introduction}

Let $\Lambda=\operatorname{PG}\left(W, \mathbb{F}_{q^{n}}\right)=\operatorname{PG}\left(1, q^{n}\right)$, where $W$ is a vector space of dimension 2 over $\mathbb{F}_{q^{n}}$. A point set $L$ of $\Lambda$ is said to be an $\mathbb{F}_{q}$-linear set of $\Lambda$ of rank $\rho$ if it

*The research was supported by the Italian National Group for Algebraic and Geometric Structures and their Applications (GNSAGA - INdAM). 
is defined by the non-zero vectors of a $\rho$-dimensional $\mathbb{F}_{q}$-vector subspace $U$ of $W$, i.e.

$$
L=L_{U}=\left\{\langle\mathbf{u}\rangle_{\mathbb{F}_{q^{n}}}: \mathbf{u} \in U \backslash\{\mathbf{0}\}\right\} .
$$

Two linear sets $L_{U}$ and $L_{W}$ of $\mathrm{PG}\left(1, q^{n}\right)$ are said to be PCL-equivalent if there is an element $\phi$ in $\operatorname{P\Gamma L}\left(2, q^{n}\right)$ such that $L_{U}^{\phi}=L_{W}$. It may happen that two $\mathbb{F}_{q}$-linear sets $L_{U}$ and $L_{W}$ of $\mathrm{PG}\left(1, q^{n}\right)$ are PCL-equivalent even if the $\mathbb{F}_{q^{-}}$-vector subspaces $U$ and $W$ are not in the same orbit of $\Gamma L\left(2, q^{n}\right)$ (see [12] and [5] for further details).

Lunardon and Polverino in [28] (see also [26]) show that every linear set is a projection of a canonical subgeometry, where a canonical subgeometry in $\mathrm{PG}\left(m-1, q^{n}\right)$ is a linear set $L$ of rank $m$ such that $\langle L\rangle=\operatorname{PG}\left(m-1, q^{n}\right)$ (1). In particular, by [28, Theorems 1 and 2] (adapted to the projective line case), for each $\mathbb{F}_{q}$-linear set $L_{U}$ of the projective line $\Lambda=\operatorname{PG}\left(1, q^{n}\right)$ of rank $n$ there exist a canonical subgeometry $\Sigma \cong \operatorname{PG}(n-1, q)$ of $\Sigma^{*}=\operatorname{PG}\left(n-1, q^{n}\right)$, and an $(n-3)$-subspace $\Gamma$ of $\Sigma^{*}$ disjoint from $\Sigma$ and from $\Lambda$ such that

$$
L_{U}=\mathrm{p}_{\Gamma, \Lambda}(\Sigma)=\{\langle\Gamma, P\rangle \cap \Lambda: P \in \Sigma\} .
$$

We call $\Gamma$ and $\Lambda$ the vertex (or center) and the axis of the projection, respectively.

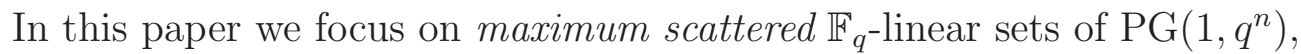
that is, $\mathbb{F}_{q}$-linear sets of rank $n$ in $\mathrm{PG}\left(1, q^{n}\right)$ of size $\left(q^{n}-1\right) /(q-1)$. In this case, we also say that the related $\mathbb{F}_{q}$-subspace is maximum scattered. Recall that the weight of a point $P=\langle\mathbf{u}\rangle_{\mathbb{F}_{q^{n}}}$ is $w_{L_{U}}(P)=\operatorname{dim}_{\mathbb{F}_{q}}\left(U \cap\langle\mathbf{u}\rangle_{\mathbb{F}_{q^{n}}}\right)$. A linear set $L_{U}$ is scattered if and only if each of its points has weight one.

If $\langle(0,1)\rangle_{\mathbb{F}_{q^{n}}}$ is not contained in the linear set $L_{U}$ of rank $n$ of $\mathrm{PG}\left(1, q^{n}\right)$ (which we can always assume after a suitable projectivity), then $U=U_{f}:=$ $\left\{(x, f(x)): x \in \mathbb{F}_{q^{n}}\right\}$ for some linearized polynomial (or q-polynomial) $f(x)=$ $\sum_{i=0}^{n-1} a_{i} x^{q^{i}} \in \mathbb{F}_{q^{n}}[x]$. In this case we will denote the associated linear set by $L_{f}$.

The first example of maximum scattered $\mathbb{F}_{q}$-linear set, found by Blokhuis and Lavrauw in [2], is known as linear sets of pseudoregulus type and can be defined (see [25, Section 4]) as any linear set PГL-equivalent to

$$
L^{1}=\left\{\left\langle\left(x, x^{q}\right)\right\rangle_{\mathbb{F}_{q^{n}}}: x \in \mathbb{F}_{q^{n}}^{*}\right\} .
$$

\footnotetext{
${ }^{1}$ Angle brackets without the indication of a field will denote the projective span of a set of points in a projective space.
} 
A characterization of the linear sets of pseudoregulus type has been given by Csajbók and Zanella in [13] as particular projections of a canonical subgeometry (see Theorem 1.1).

Theorem 1.1. [13, Theorem 2.3] Let $\Sigma$ be a canonical subgeometry of PG $(n-$ $\left.1, q^{n}\right), q>2, n \geq 3$. Assume that $\Gamma$ and $\Lambda$ are an $(n-3)$-subspace and a line of $\mathrm{PG}\left(n-1, q^{n}\right)$, respectively, such that $\Sigma \cap \Gamma=\Lambda \cap \Gamma=\emptyset$. Then the following assertions are equivalent:

1. The set $\mathrm{p}_{\Gamma, \Lambda}(\Sigma)$ is a scattered $\mathbb{F}_{q}$-linear set of pseudoregulus type;

2. A generator $\hat{\sigma}$ exists of the subgroup of $\operatorname{P\Gamma L}\left(n, q^{n}\right)$ fixing $\Sigma$ pointwise, such that $\operatorname{dim}\left(\Gamma \cap \Gamma^{\hat{\sigma}}\right)=n-4$; furthermore $\Gamma$ is not contained in the span of any hyperplane of $\Sigma$;

3. There exists a point $P_{\Gamma}$ and a generator $\hat{\sigma}$ of the subgroup of $\operatorname{P\Gamma L}\left(n, q^{n}\right)$ fixing $\Sigma$ pointwise, such that $\left\langle P_{\Gamma}, P_{\Gamma}^{\hat{\sigma}}, \ldots, P_{\Gamma}^{\hat{\sigma}^{n-1}}\right\rangle=\mathrm{PG}\left(n-1, q^{n}\right)$, and

$$
\Gamma=\left\langle P_{\Gamma}, P_{\Gamma}^{\hat{\sigma}}, \ldots, P_{\Gamma}^{\hat{\sigma}^{n-3}}\right\rangle \text {. }
$$

Few other families of maximum scattered linear sets of $\mathrm{PG}\left(1, q^{n}\right)$ are known, see [7, 11]. We will deal with the only remaining family of maximum scattered linear sets existing for each value of $n$. Such a family has been introduced by Lunardon and Polverino in [27] for $s=1$ and generalized by Sheekey in [37] and is defined as follows

$$
L_{s, \delta}^{n}=\left\{\left\langle\left(x, \delta x^{q^{s}}+x^{q^{n-s}}\right)\right\rangle_{\mathbb{F}_{q^{n}}}: x \in \mathbb{F}_{q^{n}}^{*}\right\},
$$

with $n \geq 4, \mathrm{~N}_{q^{n} / q}(\delta) \notin\{0,1\}(2)$ and $(s, n)=1$. More generally, we will call each linear set equivalent to a maximum scattered linear set of the form (11), with $\delta \neq 0$, of Lunardon-Polverino type (or shortly LP-type). For some values of $s, \delta$ and $n, \mathrm{~N}_{q^{n} / q}(\delta) \notin\{0,1\}$ is a necessary condition for $L_{s, \delta}^{n}$ to be scattered, see Section 4. Up to our knowledge, no scattered $L_{s, \delta}^{n}$ is known satisfying $\mathrm{N}(\delta)=1$.

Our aim is to prove characterizations of maximum scattered linear sets of LP-type in the spirit of the characterization of the linear sets of pseudoregulus type, cf. Theorem 1.1. As a consequence, we will construct new examples of maximum scattered linear sets in $\mathrm{PG}\left(1, q^{6}\right)$. As showed in [37, Sect. 5],

\footnotetext{
${ }^{2}$ This condition implies $q \neq 2$.
} 
this also yields to new examples of MRD-codes in $\mathbb{F}_{q}^{6 \times 6}$ with left idealiser isomorphic to $\mathbb{F}_{q^{6}}[7$, Proposition 6.1] (see also [9, 10, 38]), see last section for more details on the connections with MRD-codes.

We will work in the following framework. Let $x_{0}, \ldots, x_{n-1}$ be the homogeneous coordinates of $\mathrm{PG}\left(n-1, q^{n}\right)$ and let

$$
\Sigma=\left\{\left\langle\left(x, x^{q}, \ldots, x^{q^{n-1}}\right)\right\rangle_{\mathbb{F}_{q^{n}}}: x \in \mathbb{F}_{q^{n}}\right\}
$$

be a fixed canonical subgeometry of $\operatorname{PG}\left(n-1, q^{n}\right)$. The collineation $\hat{\sigma}$ of $\mathrm{PG}\left(n-1, q^{n}\right)$ defined by $\left\langle\left(x_{0}, \ldots, x_{n-1}\right)\right\rangle_{\mathbb{F}_{q^{n}}}^{\hat{\sigma}}=\left\langle\left(x_{n-1}^{q}, x_{0}^{q}, \ldots, x_{n-2}^{q}\right)\right\rangle_{\mathbb{F}_{q^{n}}}$ fixes precisely the points of $\Sigma$. Note that if $\sigma$ is a collineation of $\operatorname{PG}\left(n-1, q^{n}\right)$ such that $\operatorname{Fix}(\sigma)=\Sigma$, then $\sigma=\hat{\sigma}^{s}$, with $(s, n)=1$.

\section{Possible configurations of the vertex of the projection}

Following [19, Section 3], we are able to describe the structure of the vertex of the projection, under certain assumptions regarding the dimension of the intersections with some of its conjugates w.r.t. a collineation of $\mathrm{PG}\left(n-1, q^{n}\right)$ fixing the chosen subgeometry pointwise.

We start by recalling the following lemma.

Lemma 2.1. [23, Lemma 3] If $S$ is a nonempty projective subspace of $d i$ mension $k$ of $\mathrm{PG}\left(n-1, q^{n}\right)$ fixed by $\sigma$, then $S$ meets $\Sigma$ in an $\mathbb{F}_{q^{-}}$-subspace of dimension $k$. In particular, $S \cap \Sigma \neq \emptyset$.

Since the vertex of the projection is disjoint from $\Sigma$, we have that $\operatorname{dim}(\Gamma \cap$ $\left.\Gamma^{\sigma}\right) \leq \operatorname{dim} \Gamma-1$. We characterize the extremal case, i.e. when $\operatorname{dim}\left(\Gamma \cap \Gamma^{\sigma}\right)=$ $\operatorname{dim} \Gamma-1$.

Theorem 2.2. Let $\Gamma$ be a subspace of $\mathrm{PG}\left(n-1, q^{n}\right)$ of dimension $k$ and such that $\Gamma \cap \Sigma=\emptyset$. If $\operatorname{dim}\left(\Gamma \cap \Gamma^{\sigma}\right)=k-1$, then there exists exactly one point $P$ in $\mathrm{PG}\left(n-1, q^{n}\right)$ such that

$$
\Gamma=\left\langle P, P^{\sigma}, \ldots, P^{\sigma^{k}}\right\rangle
$$

Furthermore, $P^{\sigma^{n-1}} \notin \Gamma$. 
Proof. The hypotheses imply $k \geq 0$. For $k=0, \Gamma$ is a point $P$. If $P^{\sigma^{n-1}} \in \Gamma$, then $P \in \Sigma$, a contradiction. The remaining statements are trivial for this $k$.

Now suppose that the assertion is true for $(k-1)$-dimensional subspaces, and $k \geq 1$. Let denote by $\Omega=\Gamma \cap \Gamma^{\sigma}$. Clearly, $\left\langle\Omega, \Omega^{\sigma}\right\rangle \subseteq \Gamma^{\sigma}$ and $\operatorname{dim} \Gamma^{\sigma}=k$. By our assumption, $\operatorname{dim} \Omega=k-1$ and also $\operatorname{dim}\left(\Omega \cap \Omega^{\sigma}\right)=k-2$. Indeed,

$$
\operatorname{dim}\left(\Omega \cap \Omega^{\sigma}\right)=2(k-1)-\operatorname{dim}\left\langle\Omega, \Omega^{\sigma}\right\rangle \geq 2 k-2-k=k-2 .
$$

So,

$$
k-2 \leq \operatorname{dim}\left(\Omega \cap \Omega^{\sigma}\right) \leq k-1,
$$

and since $\Omega \neq \Omega^{\sigma}$, otherwise by Lemma 2.1 we should have $\Gamma \cap \Sigma \neq \emptyset$, we get $\operatorname{dim}\left(\Omega \cap \Omega^{\sigma}\right)=k-2$. Therefore, there exists a point $P^{\prime} \in \Omega$ such that

$$
\Omega=\Gamma \cap \Gamma^{\sigma}=\left\langle P^{\prime}, P^{\prime \sigma}, \ldots, P^{\prime \sigma^{k-1}}\right\rangle .
$$

By induction hypothesis, $P^{\prime \sigma^{n-1}} \notin \Gamma \cap \Gamma^{\sigma}$. So,

$$
\Gamma=\left\langle P, P^{\sigma}, \ldots, P^{\sigma^{k}}\right\rangle
$$

with $P=P^{\prime \sigma^{n-1}}$.

Regarding the uniqueness, if $\Gamma=\left\langle Q, Q^{\sigma}, \ldots, Q^{\sigma^{k}}\right\rangle$ for some point $Q$, then $\Omega=\left\langle Q^{\sigma}, \ldots, Q^{\sigma^{k}}\right\rangle$. By induction, this implies $Q^{\sigma}=P^{\prime}$ above defined, and $Q=P$.

Finally note that $P^{\sigma^{n-1}} \in \Gamma$ would imply $\Gamma^{\sigma^{n-1}}=\Gamma$ and $\Gamma \cap \Sigma \neq \emptyset$, a contradiction.

The next result follows for $r=1$ from Theorem 2.2 .

Theorem 2.3. Let $\Gamma$ be a subspace of $\mathrm{PG}\left(n-1, q^{n}\right)$ of dimension $k \geq 0$ such that $\Gamma \cap \Sigma=\emptyset$, and $\operatorname{dim}\left(\Gamma \cap \Gamma^{\sigma}\right) \geq k-2$. Let $r$ be the least positive integer satisfying the condition

$$
\operatorname{dim}\left(\Gamma \cap \Gamma^{\sigma} \cap \Gamma^{\sigma^{2}} \cap \ldots \cap \Gamma^{\sigma^{r}}\right)>k-2 r .
$$

Then there is a point $P \in \mathrm{PG}(n-1, q)$ satisfying

(i) $P, P^{\sigma}, \ldots, P^{\sigma^{k-r+1}}$ are independent points contained in $\Gamma$;

(ii) $P^{\sigma^{n-1}} \notin \Gamma$. 
If $r<(k+2) / 2$, then the point $P$ satisfying conditions (i) and (ii) is unique.

We will call the integer $r$ of the above statement the intersection number of $\Gamma$ w.r.t. $\sigma$ and we will denote it by $\operatorname{intn}_{\sigma}(\Gamma)$.

Proof. Preliminary remarks. Since $\sigma$ is a collineation and since $\operatorname{dim}\left(\Gamma \cap \Gamma^{\sigma}\right) \geq$ $k-2$, for any positive integer $t$ it holds

$$
\begin{gathered}
\operatorname{dim}\left(\Gamma \cap \Gamma^{\sigma} \cap \ldots \cap \Gamma^{\sigma^{t+1}}\right)= \\
\operatorname{dim} \Gamma+\operatorname{dim}\left(\Gamma^{\sigma} \cap \ldots \cap \Gamma^{\sigma^{t+1}}\right)-\operatorname{dim}\left(\left\langle\Gamma \cup\left(\Gamma^{\sigma} \cap \ldots \cap \Gamma^{\sigma^{t+1}}\right)\right\rangle\right) \geq \\
\operatorname{dim} \Gamma+\operatorname{dim}\left(\Gamma \cap \ldots \cap \Gamma^{\sigma^{t}}\right)-\operatorname{dim}\left(\left\langle\Gamma \cup \Gamma^{\sigma}\right\rangle\right) \geq \operatorname{dim}\left(\Gamma \cap \ldots \cap \Gamma^{\sigma^{t}}\right)-2 .
\end{gathered}
$$

This implies $\operatorname{dim}\left(\Gamma \cap \ldots \cap \Gamma^{\sigma^{t}}\right)=k-2 t$ for any $0 \leq t<r$; so, taking $t=r-1$, $k-2(r-1) \geq-1$, that is $r \leq(k+3) / 2$. Furthermore, if $\operatorname{dim}\left(\Gamma \cap \ldots \cap \Gamma^{\sigma^{t}}\right) \geq 0$, then

$$
\operatorname{dim}\left(\Gamma \cap \Gamma^{\sigma} \cap \ldots \cap \Gamma^{\sigma^{t+1}}\right) \leq \operatorname{dim}\left(\Gamma \cap \ldots \cap \Gamma^{\sigma^{t}}\right)-1,
$$

for otherwise $\Sigma \cap \Gamma \cap \ldots \cap \Gamma^{\sigma^{t}} \neq \emptyset$. This implies

$$
\operatorname{dim}\left(\Gamma \cap \Gamma^{\sigma} \cap \Gamma^{\sigma^{2}} \cap \ldots \cap \Gamma^{\sigma^{r}}\right)=k-2 r+1 \quad \text { for } r \neq \frac{k+3}{2} .
$$

Note that for $r=\frac{k+3}{2}$ then $\operatorname{dim}\left(\Gamma \cap \Gamma^{\sigma} \cap \Gamma^{\sigma^{2}} \cap \ldots \cap \Gamma^{\sigma^{r}}\right)=k-2 r+2=-1$.

Existence of $P$, by induction on $r$. For $r=1$, the assertion follows from Theorem 2.2. Assume then that Theorem 2.3 holds (except possibly for the uniqueness part) for $r-1$, and $r \geq 2$. Let $\Omega=\Gamma \cap \Gamma^{\sigma}$ and $\operatorname{dim} \Omega=k-2=: k^{\prime}$. If $k^{\prime}=-1$, then the thesis is trivial.

Now suppose $k^{\prime} \geq 0$. Then it holds

$$
\operatorname{dim}\left(\Omega \cap \ldots \cap \Omega^{\sigma^{t}}\right)=k^{\prime}-2 t
$$

for $t<r-1$, whereas

$$
\operatorname{dim}\left(\Omega \cap \ldots \cap \Omega^{\sigma^{r-1}}\right)>k^{\prime}-2(r-1)=k-2 r .
$$

By induction hypothesis there is a point $P^{\prime} \in \mathrm{PG}\left(n-1, q^{n}\right)$ satisfying

(A) $P^{\prime}, P^{\prime \sigma}, \ldots, P^{\prime \sigma^{k^{\prime}-(r-1)+1}}=P^{\prime \sigma^{k-r}}$ are independent points;

(B) $P^{\prime}, P^{\prime \sigma}, \ldots, P^{\prime \sigma^{k-r}} \in \Omega$; 
(C) $P^{\prime \sigma^{n-1}} \notin \Omega$.

Let $P=P^{\prime \sigma^{n-1}}$. Then $(\mathrm{B})$ implies that $P, P^{\sigma}, \ldots, P^{\sigma^{k-r+1}}$ are points contained in $\Gamma$; both $(\mathrm{C})$ and $(\mathrm{A})$ imply that they are independent. $P^{\sigma^{n-1}} \in \Gamma$ would imply

$$
P^{\sigma^{r-2}}, P^{\sigma^{r-1}}, \ldots, P^{\sigma^{k-r+1}} \in \Gamma \cap \Gamma^{\sigma} \cap \ldots \cap \Gamma^{\sigma^{r-1}}
$$

contradicting $\operatorname{dim}\left(\Gamma \cap \Gamma^{\sigma} \cap \ldots \cap \Gamma^{\sigma^{r-1}}\right)=k-2 r+2$.

Uniqueness of $P$. By the previous considerations it follows that there exists at least one point $P$ such that $P, P^{\sigma}, \ldots, P^{\sigma^{k-r+1}}$ are independent points contained in $\Gamma$. From (44) it follows that

$$
\Lambda:=\Gamma \cap \Gamma^{\sigma} \cap \Gamma^{\sigma^{2}} \cap \ldots \cap \Gamma^{\sigma^{r}}=\left\langle P^{\sigma^{r}}, \ldots, P^{\sigma^{k-r+1}}\right\rangle,
$$

has dimension $k-2 r+1>-1$. Furthermore, $\operatorname{dim}\left(\Lambda \cap \Lambda^{\sigma}\right)=k-2 r$, otherwise $\Gamma \cap \Sigma \neq \emptyset$. It follows that $\Lambda$ satisfies the hypotheses of Theorem 2.2 and hence the point $P$ is unique.

Remark 2.4. It is clear that $P$ is as in the previous result, it follows that

$$
\operatorname{dim} L(P) \geq k-r+2,
$$

where $L(P)=\left\langle P, P^{\sigma}, \ldots, P^{\sigma^{n-1}}\right\rangle$.

Remark 2.5. A similar idea to the intersection number for a vertex of a linear set has been presented in [34] (see also [19, 35]), where the authors used sequences of the dimensions of certain intersections as invariants for rank metric codes. See also the last section.

\section{Characterization of linear sets of LP-type}

\subsection{Sufficient conditions}

We are now ready to state sufficient conditions for a linear set to be of LPtype. In the following we denote by $\mathrm{N}(-)$ the norm over $\mathbb{F}_{q}$, for short.

Theorem 3.1. In $\mathrm{PG}\left(n-1, q^{n}\right), n \geq 4$, let $\Gamma$ be a subspace of dimension $n-3, \Lambda$ a line, and $\Sigma \cong \operatorname{PG}(n-1, q)$ a canonical subgeometry, such that $\Gamma \cap \Sigma=\emptyset=\Gamma \cap \Lambda$. Assume $L=\mathrm{p}_{\Gamma, \Lambda}(\Sigma)$ is a scattered linear set of $\Lambda$. If 
$\operatorname{intn}_{\sigma}(\Gamma)=2$ for some generator $\sigma$ of the subgroup of $\operatorname{P\Gamma L}\left(n, q^{n}\right)$ fixing $\Sigma$ pointwise, then there exists a unique point $P$ such that

$$
\Gamma=\left\langle P, P^{\sigma}, \ldots, P^{\sigma^{n-4}}, Q\right\rangle .
$$

Furthermore, if the line $\left\langle P^{\sigma^{n-1}}, P^{\sigma^{n-3}}\right\rangle$ meets $\Gamma$, then $L$ is of LP-type.

Proof. An integer $s$ exists such that $(s, n)=1$ and $\sigma=\hat{\sigma}^{s}$, i.e. the $i$-th component (3) of $\left\langle\left(x_{0}, x_{1}, \ldots, x_{n-1}\right)\right\rangle_{\mathbb{F}_{q^{n}}}^{\sigma}$ is $x_{i+s}^{q^{s}}$, where $i+s$ is seen modulo $n$. By Theorem 2.3 , there exist $P$ and $Q$ in $\Gamma$ such that

$$
\Gamma=\left\langle P, P^{\sigma}, \ldots, P^{\sigma^{n-4}}, Q\right\rangle
$$

Denote by $R=P^{\sigma^{n-2}}$, then

$$
\Gamma=\left\langle R^{\sigma^{2}}, R^{\sigma^{3}}, \ldots, R^{\sigma^{n-2}}, Q\right\rangle
$$

and $Q$ may be chosen in $\left\langle R^{\sigma}, R^{\sigma^{n-1}}\right\rangle$. If $\operatorname{dim}\left\langle R, R^{\sigma}, \ldots, R^{\sigma^{n-1}}\right\rangle<n-1$, then, since $Q \in\left\langle R^{\sigma}, R^{\sigma^{n-1}}\right\rangle$, it follows that

$$
\Gamma \subseteq\left\langle R, R^{\sigma}, \ldots, R^{\sigma^{n-1}}\right\rangle
$$

i.e. $\Gamma$ is contained in a subspace fixed by $\sigma$ of dimension either $n-3$ or $n-2$. In both the cases we get a contradiction because of $\operatorname{intn}_{\sigma}(\Gamma)=2$. So, $\operatorname{dim}\left\langle R, R^{\sigma}, \ldots, R^{\sigma^{n-1}}\right\rangle=n-1$, and by [3, Proposition 3.1] there exists a linear collineation $\mathbf{k}$ fixing $\Sigma$ such that $R^{\mathbf{k}}=\langle(1,0, \ldots, 0)\rangle_{\mathbb{F}_{q^{n}}}$. Clearly, $\Gamma^{\mathbf{k}}$ satisfies the same hypothesis as $\Gamma$, since $\mathbf{k}$ and $\sigma$ commute. For these reasons, we may assume that $R=\langle(1,0, \ldots, 0)\rangle_{\mathbb{F}_{q^{n}}}$. In particular, it follows that the coordinates of $R^{\sigma^{i}}$ are $\mathbf{e}_{i s}(\bmod n)$, where $\mathbf{e}_{j}$ is the vector whose $j$-th component is one and all the others are zero. And by hypothesis we may assume that $Q=\left\langle\mathbf{e}_{s}-\delta \mathbf{e}_{s(n-1)}\right\rangle_{\mathbb{F}_{q^{n}}}$. Hence we can choose as $\Lambda=\left\langle R, R^{\sigma^{n-1}}\right\rangle$, so $\Gamma$ has equations $x_{0}=0, x_{s(n-1)}=-\delta x_{s}$, and $\Lambda$ is defined by $x_{i}=0$ for $i \in\{s, \ldots, s(n-2)\}$.

Therefore,

$$
L=\mathrm{p}_{\Gamma, \Lambda}(\Sigma) \simeq\left\{\left\langle\left(x, \delta x^{q^{s}}+x^{q^{s(n-1)}}\right)\right\rangle_{\mathbb{F}_{q^{n}}}: x \in \mathbb{F}_{q^{n}}\right\},
$$

i.e. $L$ is of LP-type.

\footnotetext{
${ }^{3}$ Starting to count from zero.
} 
Each linear set of LP-type $L_{s, \delta}^{n}$ (1) of $\mathrm{PG}\left(1, q^{n}\right)$, with $n \geq 4$ and $(s, n)=1$, can be realized as the projection of $\Sigma$ choosing $\Gamma$ and $\Lambda$ as follows

$$
\Gamma:\left\{\begin{array}{l}
x_{0}=0 \\
x_{s(n-1)}=-\delta x_{s}
\end{array} \quad \text { and } \Lambda: x_{i}=0, \quad i \in\{s, \ldots, s(n-2)\} .\right.
$$

Therefore, as a direct consequence of Theorem 3.1 we provide a characterization result of linear sets of LP-type.

Theorem 3.2. Let $\Sigma$ be a canonical subgeometry of $\mathrm{PG}\left(n-1, q^{n}\right), q>2$ and $n \geq 4$. Let $L$ be a scattered linear set in $\Lambda=\operatorname{PG}\left(1, q^{n}\right)$. Then $L$ is a linear set of LP-type if and only if

(i) there exists an $(n-3)$-subspace $\Gamma$ of $\mathrm{PG}\left(n-1, q^{n}\right)$ such that $\Gamma \cap \Sigma=$ $\Gamma \cap \Lambda=\emptyset$ and $L=\mathrm{p}_{\Gamma, \Lambda}(\Sigma)$;

(ii) there exists a generator $\sigma$ of the subgroup of $\operatorname{P\Gamma L}\left(n, q^{n}\right)$ fixing $\Sigma$ pointwise, such that $\operatorname{int}_{\sigma}(\Gamma)=2$;

(iii) there exist a unique point $P \in \mathrm{PG}\left(n-1, q^{n}\right)$ and some point $Q$ such that

$$
\Gamma=\left\langle P, P^{\sigma}, \ldots, P^{\sigma^{n-4}}, Q\right\rangle
$$

(iv) the line $\left\langle P^{\sigma^{n-1}}, P^{\sigma^{n-3}}\right\rangle$ meets $\Gamma$.

\subsection{Necessary conditions}

Very recently, Csajbók, Marino and Polverino in [5] have investigated the equivalence problem between $\mathbb{F}_{q}$-linear sets of rank $n$ on the projective line $\operatorname{PG}\left(1, q^{n}\right)$. The idea is first to study the $\Gamma \mathrm{L}\left(2, q^{n}\right)$-orbits of the subspace $U$ defining the linear set $L_{U}$ and then to study the equivalence between two linear sets. More precisely, they give the following definition of $\Gamma$ L-class (see [5. Definitions 2.5]) of an $\mathbb{F}_{q}$-linear set of a line.

Let $L_{U}$ be an $\mathbb{F}_{q}$-linear set of $\mathrm{PG}\left(V, \mathbb{F}_{q^{n}}\right)=\operatorname{PG}\left(1, q^{n}\right)$ of rank $n$ with maximum field of linearity $\mathbb{F}_{q}(4)$.

We say that $L_{U}$ is of $\Gamma \mathrm{L}$-class $s$ if $s$ is the greatest integer such that there exist $\mathbb{F}_{q}$-subspaces $U_{1}, \ldots, U_{s}$ of $V$ with $L_{U_{i}}=L_{U}$ for $i \in\{1, \ldots, s\}$ and there is no $f \in \Gamma \mathrm{L}\left(2, q^{n}\right)$ such that $U_{i}=U_{j}^{f}$ for each $i \neq j, i, j \in\{1,2, \ldots, s\}$.

\footnotetext{
${ }^{4}$ The maximum field of linearity of an $\mathbb{F}_{q^{-}}$-linear set $L_{U}$ as $\mathbb{F}_{q^{\ell}}$ if $\ell$ is the largest integer such that $\ell \mid n$ and $L_{U}$ is an $\mathbb{F}_{q^{\ell}}$-linear set.
} 
If $L_{U}$ is of $\Gamma$-class one, then $L_{U}$ is said to be simple, i.e. when the $\Gamma \mathrm{L}\left(2, q^{n}\right)$-orbit of $U$ completely determine the $\mathrm{P} \Gamma \mathrm{L}\left(2, q^{n}\right)$-orbit of $L_{U}$. For $n \leq 4$, any linear set in $\mathrm{PG}\left(1, q^{n}\right)$ is simple [5, Theorem 4.5].

The $\Gamma$ L-class of a linear set is a projective invariant (by [5, Proposition 2.6]) and play a crucial role in the study of linear sets up to equivalences. Using these notions and by developing some new techniques, the authors in [6] prove that in $\operatorname{PG}\left(1, q^{5}\right)$ each $\mathbb{F}_{q}$-linear set $L_{f}$ of rank 5 and with maximum field of linearity $\mathbb{F}_{q}$ is of $\Gamma$ L-class at most 2, proving also that if $L_{U}$ is equivalent to $L_{f}$ then $U$ is $\Gamma L$-equivalent to either $U_{f}$ or to $U_{f}^{\perp}=U_{\hat{f}}$, where the non-degenerate symmetric bilinear form of $\mathbb{F}_{q^{n}}$ over $\mathbb{F}_{q}$ defined by

$$
\langle x, y\rangle=\operatorname{Tr}_{q^{n} / q}(x y),
$$

for each $x, y \in \mathbb{F}_{q^{n}}$ is taken into account. Then the adjoint $\hat{f}$ of the linearized polynomial $f(x)=\sum_{i=0}^{n-1} a_{i} x^{q^{i}} \in \tilde{\mathcal{L}}_{n, q}$ with respect to the bilinear form $\langle$,$\rangle is$

$$
\hat{f}(x)=\sum_{i=0}^{n-1} a_{i}^{q^{n-i}} x^{q^{n-i}}
$$

i.e.

$$
\operatorname{Tr}_{q^{n} / q}(x f(y))=\operatorname{Tr}_{q^{n} / q}(y \hat{f}(x)),
$$

for each $x, y \in \mathbb{F}_{q^{n}}$.

For linear sets of LP-type the following is known.

Theorem 3.3. [6, 11] A maximum scattered linear set of LP-type

$$
L_{s, \delta}^{n}=L_{f}=\left\{\left\langle\left(x, \delta x^{q^{s}}+x^{q^{n-s}}\right)\right\rangle_{\mathbb{F}^{n}}: x \in \mathbb{F}_{q^{n}}\right\} \subseteq \mathrm{PG}\left(1, q^{n}\right),
$$

with $(s, n)=1$ and $f(x)=\delta x^{q^{s}}+x^{q^{n-s}}$, is of $\Gamma$ L-class less than or equal to 2 for $n \in\{5,6,8\}$. Furthermore, $L_{U}$ is equivalent to $L$ if and only if $U$ is $\Gamma \mathrm{L}\left(2, q^{n}\right)$-equivalent to either $U_{f}$ or to $U_{\hat{f}}^{\perp}=U_{\hat{f}}$.

Furthermore, in [5, 40, it has been shown that there are maximum scattered linear sets of LP-type of both CL-classes one and two.

For our purpose it is important to look to the $\Gamma$ L-class in a more geometric way. The following result has been stated in [5, Section 5.2] as a consequence of [12, Theorems $6 \& 7$ ]. 
Theorem 3.4. The $\Gamma \mathrm{L}$-class of $L_{U}$ is the number of orbits in $\operatorname{Stab}(\Sigma)$ of $(n-3)$-subspaces of $\mathrm{PG}\left(n-1, q^{n}\right)$ containing $a \Gamma$ disjoint from $\Sigma$ and from $\Lambda$ such that $\mathrm{p}_{\Gamma, \Lambda}(\Sigma)$ is equivalent to $L_{U}$.

As a consequence of Theorems 3.3 and 3.4, we have the following characterization for linear sets of LP-type.

Theorem 3.5. Let $L$ be a maximum scattered linear set in $\Lambda=\mathrm{PG}\left(1, q^{n}\right)$ with $n \leq 6$ or $n=8$. Then $L$ is a linear set of LP-type if and only if for each $(n-3)$-subspace $\Gamma$ of $\mathrm{PG}\left(n-1, q^{n}\right)$ such that $L=\mathrm{p}_{\Gamma, \Lambda}(\Sigma)$, the following holds:

(i) there exists a generator $\sigma$ of the subgroup of $\mathrm{P} \Gamma \mathrm{L}\left(n, q^{n}\right)$ fixing $\Sigma$ pointwise, such that $\operatorname{intn}_{\sigma}(\Gamma)=2$;

(ii) if $P$ is the unique point of $\mathrm{PG}\left(n-1, q^{n}\right)$ such that

$$
\Gamma=\left\langle P, P^{\sigma}, \ldots, P^{\sigma^{n-4}}, Q\right\rangle,
$$

then the line $\left\langle P^{\sigma^{n-1}}, P^{\sigma^{n-3}}\right\rangle$ meets $\Gamma$.

Proof. Because of Theorem [3.3 and [5, Theorem 4.5], if $n \leq 6$ or $n=8$, then the two (possibly) not $\Gamma \mathrm{L}\left(2, q^{n}\right)$-equivalent representation for a linear set of LP-type (1) are

$$
U_{\delta x^{q^{s}}+x^{q^{n-s}}} \text { and } U_{\delta q^{n-s} x^{q^{n-s}}+x^{q^{s}}} .
$$

Therefore, by Theorem 3.4 we have that all the possible vertices of the projections to obtain a linear set of LP-type satisfy the hypothesis of Theorem 3.2 and the assertion then follows.

Remark 3.6. Note that Theorem 3.5 guarantees that each vertex of the projection of a linear set of LP-type satisfies conditions ( $i)$ and (ii), whereas Theorem 3.2 asserts the existence of a vertex of the projection of a linear set of LP-type satisfying these conditions.

As we will see in Section 5, this result may turn out to be useful to construct new examples of maximum scattered linear sets in $\mathrm{PG}\left(1, q^{n}\right)$. 


\section{A purely geometric description for odd $n$}

The next lemma proves that, for $n$ odd, the only scattered linear sets of LP-type are exactly those described by Lunardon and Polverino in [27] and Sheekey [37].

Lemma 4.1. Let $L:=\left\{\left\langle\left(x, \delta x^{q^{s}}+x^{q^{n-s}}\right)\right\rangle_{\mathbb{F}_{q^{n}}}: x \in \mathbb{F}_{q^{n}}\right\} \subseteq \operatorname{PG}\left(1, q^{n}\right)$, with $(s, n)=1$, and let $n>3$ be odd. Then $L$ is scattered if and only if $\mathrm{N}(\delta) \neq 1$.

Proof. We only have to prove that if $\mathrm{N}(\delta)=1$, then $L$ cannot be scattered. The linear set $L$ is scattered if and only if in the following set of polynomials

$$
A=\left\{\alpha x+\delta x^{q^{s}}+x^{q^{n-s}}: \alpha \in \mathbb{F}_{q^{n}}\right\}
$$

there are no polynomials with more than $q$ roots, for otherwise there would be a point $\langle(1,-\alpha)\rangle_{\mathbb{F}_{q^{n}}}$ of weight greater than one. Equivalently in the following set of polynomials

$$
A^{\prime}=\left\{f_{\alpha}(x)=\delta^{-1} x+\alpha x^{q^{s}}+x^{q^{2 s}}: \alpha \in \mathbb{F}_{q^{n}}\right\}
$$

there are no polynomials with more than $q$ roots. For any $\xi \in \mathbb{F}_{q^{n}}^{*}$ with $\mathrm{N}(\xi)=1$, the polynomial

$$
\frac{f_{\alpha}(x) \circ \xi x}{\xi^{q^{2 s}}}=\delta^{-1} \xi^{1-q^{2 s}} x+\alpha \xi^{q^{s}-q^{2 s}} x^{q^{s}}+x^{q^{2 s}}
$$

has the same number of roots of $f_{\alpha}(x)$. Note that since $n$ is odd, for any $m \in \mathbb{F}_{q^{n}}$ such that $\mathrm{N}(m)=1$ there is $\xi \in \mathbb{F}_{q^{n}}$ such that $m=\xi^{1-q^{2 s}}$. Taking into account $\mathrm{N}(\delta)=1$, this implies that for any polynomial of the form $P(x)=\gamma x+\beta x^{q^{s}}+x^{q^{2 s}}$, with $\gamma, \beta \in \mathbb{F}_{q^{n}}$ and $\mathrm{N}(\gamma)=1$, there are $\alpha$ and $\xi \in \mathbb{F}_{q^{n}}$ such that (5) coincides with $P(x)$. This is a contradiction, since there exist polynomials of type $\gamma x+\beta x^{q^{s}}+x^{q^{2 s}}, \mathrm{~N}(\gamma)=1$, with $q^{2}$ roots, e.g.

$$
\frac{1}{u^{q^{s}} v^{q^{2 s}}-u^{q^{2 s}} v^{q^{s}}} \operatorname{det}\left(\begin{array}{ccc}
x & x^{q^{s}} & x^{q^{2 s}} \\
u & u^{q^{s}} & u^{q^{2 s}} \\
v & v^{q^{s}} & v^{q^{2 s}}
\end{array}\right)
$$

where $u, v \in \mathbb{F}_{q^{n}}$ are $\mathbb{F}_{q^{-}}$-linearly independent.

The previous lemma was already proved for $n=4$ in [14] and for $s=1$ in [1]. 
Theorem 4.2. Let $\Gamma$ be a subspace of $\mathrm{PG}\left(n-1, q^{n}\right)$, $n$ odd, of dimension $n-3 \geq 2$, and $\Sigma \cong \operatorname{PG}(n-1, q)$ a canonical subgeometry of $\mathrm{PG}\left(n-1, q^{n}\right)$, such that $\Gamma \cap \Sigma=\emptyset$. Assume that a generator $\sigma$ exists of the subgroup of $\operatorname{P\Gamma L}\left(n, q^{n}\right)$ fixing $\Sigma$ pointwise, such that $\operatorname{int}_{\sigma}(\Gamma)=2$. Then there exists a point $R \in \mathrm{PG}\left(n-1, q^{n}\right)$ such that

$$
R^{\sigma^{2}}, R^{\sigma^{3}}, \ldots, R^{\sigma^{n-2}} \in \Gamma \text {. }
$$

Furthermore assume that $\left\langle R^{\sigma}, R^{\sigma^{n-1}}\right\rangle$ and $\Gamma$ meet in a point $Q$ and $R^{\sigma} \neq$ $Q \neq R^{\sigma^{n-1}}$. Let $Q^{\prime}$ be the point such that the pair $\left\{R^{\sigma}, R^{\sigma^{n-1}}\right\}$ separates $\left\{Q, Q^{\prime}\right\}$ harmonically. Such $Q^{\prime}$ is defined by the property that there are two representative vectors $v_{0}$ and $v_{1}$ for $R^{\sigma}$ and $R^{\sigma^{n-1}}$, respectively, such that

$\left\langle v_{0}+v_{1}\right\rangle_{\mathbb{F}_{q^{n}}}=Q,\left\langle v_{0}-v_{1}\right\rangle_{\mathbb{F}_{q^{n}}}=Q^{\prime}$. Under the assumptions above, the linear set $L=\mathrm{p}_{\Gamma, \Lambda}(\Sigma)$, with $\Lambda$ a line disjoint from $\Gamma$, is a maximum scattered linear set of LP-type if and only if

$$
\Sigma \cap\left\langle R, R^{\sigma^{2}}, R^{\sigma^{3}}, \ldots, R^{\sigma^{n-2}}, Q^{\prime}\right\rangle=\emptyset .
$$

Proof. As in Theorem 3.1 it may be assumed that the coordinates of $Q$ are $x_{s}=1, x_{s(n-1)}=-\delta \in \mathbb{F}_{q^{n}}^{*}, x_{i}=0$ otherwise. The coordinates of $Q^{\prime}$ are $x_{s}=1, x_{s(n-1)}=\delta \in \mathbb{F}_{q^{n}}^{*}, x_{i}=0$ otherwise. The span $W=$ $\left\langle R, R^{\sigma^{2}}, R^{\sigma^{3}}, \ldots, R^{\sigma^{n-2}}, Q^{\prime}\right\rangle$ and $R^{\sigma}$ are complementary subspaces of $\mathrm{PG}(n-$ $\left.1, q^{n}\right)$. So, $W$ is a hyperplane and its equation is $-\delta x_{s}+x_{s(n-1)}=0$. A point $\left\langle\left(u, u^{q}, \ldots, u^{q(n-1)}\right)\right\rangle_{\mathbb{F}_{q^{n}}}$ of $\Sigma$ belongs to $W$ if and only if $-\delta u^{q^{s}}+u^{q^{s(n-1)}}=0$, equivalent to

$$
\delta=u^{q^{s}\left(q^{s(n-2)}-1\right)} .
$$

Since $n$ is odd, $s(n-2)$ is coprime with $n$. This implies that an $u \in \mathbb{F}_{q^{n}}^{*}$ exists satisfying (17) if and only if $\mathrm{N}(\delta)=1$, which is a contradiction because of Lemma 4.1 .

\section{New constructions}

In this section we will deal with the following family of linear sets

$$
\mathcal{L}:=\left\{\left\langle\left(x, x^{q}-x^{q^{2}}+x^{q^{4}}+x^{q^{5}}\right)\right\rangle_{\mathbb{F}_{q^{6}}}: x \in \mathbb{F}_{q^{6}}^{*}\right\} \subseteq \mathrm{PG}\left(1, q^{6}\right), \quad q \equiv 1 \quad(\bmod 4) .
$$

We will show that for some choices of $q$ we may get new examples of maximum scattered linear sets. This family of linear sets can be obtained by projecting 
the canonical subgeometry $\Sigma=\left\{\left\langle\left(x, x^{q}, x^{q^{2}}, x^{q^{3}}, x^{q^{4}}, x^{q^{5}}\right)\right\rangle_{\mathbb{F}_{q^{6}}}: x \in \mathbb{F}_{q^{6}}^{*}\right\}$ from

$$
\Gamma:\left\{\begin{array}{l}
x_{0}=0 \\
x_{5}=-x_{4}-x_{1}+x_{2}
\end{array}\right.
$$

to

$$
\Lambda:\left\{\begin{array}{l}
x_{1}=0 \\
x_{2}=0 \\
x_{3}=0 \\
x_{4}=0
\end{array}\right.
$$

Let us consider $\sigma \in \operatorname{P\Gamma L}\left(6, q^{6}\right)$ defined as

$$
\left(\left\langle\left(x_{0}, x_{1}, x_{2}, x_{2}, x_{4}, x_{5}\right)\right\rangle_{\mathbb{F}_{q^{6}}}\right)^{\sigma}=\left\langle\left(x_{5}^{q}, x_{0}^{q}, x_{1}^{q}, x_{2}^{q}, x_{3}^{q}, x_{4}^{q}\right)\right\rangle_{\mathbb{F}_{q^{6}}}
$$

and $\bar{\sigma}:=\sigma^{5}$, which are the two generators of the subgroup of $\operatorname{P\Gamma L}\left(6, q^{6}\right)$ fixing $\Sigma$ pointwise. Then

$$
\Gamma^{\sigma}:\left\{\begin{array}{l}
x_{1}=0 \\
x_{0}=-x_{5}-x_{2}+x_{3}
\end{array} \quad \text { and } \Gamma^{\sigma^{2}}:\left\{\begin{array}{l}
x_{2}=0 \\
x_{1}=-x_{0}-x_{3}+x_{4} .
\end{array}\right.\right.
$$

Therefore,

$$
\Gamma \cap \Gamma^{\sigma}:\left\{\begin{array}{l}
x_{0}=0 \\
x_{1}=0 \\
x_{4}=2 x_{2}-x_{3} \\
x_{5}=-x_{2}+x_{3}
\end{array} \quad \text { and } \quad \Gamma \cap \Gamma^{\sigma} \cap \Gamma^{\sigma^{2}}=\emptyset .\right.
$$

Hence, $\operatorname{dim}_{\mathbb{F}_{q^{6}}}\left(\Gamma \cap \Gamma^{\sigma}\right)=1$ and since $q$ is odd $\operatorname{dim}_{\mathbb{F}_{q^{6}}}\left(\Gamma \cap \Gamma^{\sigma} \cap \Gamma^{\sigma^{2}}\right)=-1$. Since $\Gamma \cap \Gamma^{\bar{\sigma}}=\left(\Gamma \cap \Gamma^{\sigma}\right)^{\sigma^{5}}$ and $\Gamma \cap \Gamma^{\bar{\sigma}} \cap \Gamma^{\bar{\sigma}^{2}}=\left(\Gamma \cap \Gamma^{\sigma} \cap \Gamma^{\sigma^{2}}\right)^{\sigma^{4}}$, we have that $\operatorname{dim}_{\mathbb{F}_{q^{6}}}\left(\Gamma \cap \Gamma^{\bar{\sigma}}\right)=1$ and $\operatorname{dim}_{\mathbb{F}_{q^{6}}}\left(\Gamma \cap \Gamma^{\bar{\sigma}} \cap \Gamma^{\bar{\sigma}^{2}}\right)=-1$. Therefore,

$$
\operatorname{intn}_{\sigma}(\Gamma)=\operatorname{intn}_{\bar{\sigma}}(\Gamma)=3
$$

This implies the non-equivalence of $\mathcal{L}$ with the linear set of pseudoregulus type and also it cannot be of LP-type because of Theorem 3.5 .

Computational results show that for $q \equiv 1(\bmod 4)$ the linear set $\mathcal{L}$ is maximum scattered for $q \leq 29$. We show that for $q \leq 17$ and $q \not \equiv 0(\bmod 5)$ it is also new. For $q \equiv 0(\bmod 5)$ we will prove in Proposition 5.5 that $\mathcal{L}$ is equivalent to the linear set defined in [11. 


\subsection{Known examples of maximum scattered linear sets in $\mathrm{PG}\left(1, q^{6}\right)$}

In order to decide whether the linear set $\mathcal{L}$ is new, we describe the known maximum scattered linear sets in $\mathrm{PG}\left(1, q^{6}\right)$.

We start by listing the non-equivalent (under the action of $\Gamma \mathrm{L}\left(2, q^{6}\right)$ ) maximum scattered subspaces of $\mathbb{F}_{q^{6}}^{2}$, i.e. subspaces defining maximum scattered linear sets.

Example 5.1. 1. $U^{1}:=\left\{\left(x, x^{q}\right): x \in \mathbb{F}_{q^{6}}\right\}$, see [2, 13];

2. $U_{\delta}^{2}:=\left\{\left(x, \delta x^{q}+x^{q^{5}}\right): x \in \mathbb{F}_{q^{6}}\right\}, \mathrm{N}_{q^{6} / q}(\delta) \notin\{0,1\}$ 5, see 27, 29, 37];

3. $U_{\delta}^{3}:=\left\{\left(x, \delta x^{q}+x^{q^{4}}\right): x \in \mathbb{F}_{q^{6}}\right\}, \mathrm{N}_{q^{6} / q^{3}}(\delta) \notin\{0,1\}$, satisfying further conditions on $\delta$ and $q$, see [7, Theorems 7.1 and 7.2] 6 ;

4. $U_{\delta}^{4}:=\left\{\left(x, x^{q}+x^{q^{3}}+\delta x^{q^{5}}\right): x \in \mathbb{F}_{q^{6}}\right\}, q$ odd and $\delta^{2}+\delta=1$, see [11, 31].

In order to simplify the notation, we will denote by $L^{1}$ and $L_{\delta}^{i}$ the $\mathbb{F}_{q^{-}}$ linear set defined by $U^{1}$ and $U_{\delta}^{i}$, respectively. Therefore, $L_{\delta}^{2}=L_{s, \delta}^{6}$ as defined in (1). We will also use the following notation: $\mathcal{U}:=U_{x^{q}-x^{q^{2}}+x^{q^{4}}+x^{q^{5}}}$.

In [11, Propositions 3.1, $4.1 \& 5.5]$ the following result has been proved.

Lemma 5.2. Let $L_{f}$ be one of the maximum scattered of $\mathrm{PG}\left(1, q^{6}\right)$ listed before. Then a linear set $L_{U}$ of $\mathrm{PG}\left(1, q^{6}\right)$ is $\mathrm{P} \Gamma \mathrm{L}$-equivalent to $L_{f}$ if and only if $U$ is $\Gamma \mathrm{L}$-equivalent either to $U_{f}$ or to $U_{\hat{f}}$. Furthermore, the linear set $L_{\delta}^{3}$ is simple.

The previous lemma includes results on linear sets of LP-type.

Remark 5.3. If $U_{f}$ is an $\mathbb{F}_{q}$-subspace of type 1. or 2. above, then $U_{\hat{f}}$ and $U_{f}$ are $\Gamma \mathrm{L}$-equivalent. By Lemma 5.2. this holds also for $\mathbb{F}_{q}$-subspaces of type 3.

\subsection{The linear set $\mathcal{L}$}

Here we deal with the equivalence issue between the linear sets defined by Example 5.1 and the linear set $\mathcal{L}$. As already noted, we just have to check the equivalence with the linear sets $L_{\delta}^{3}$ and with $L_{\delta}^{4}$ defined by the subspaces 3. and 4. in Example 5.1, because of the construction of $\mathcal{L}$ and Theorems 1.1 and 3.5 .

\footnotetext{
${ }^{5}$ This condition implies $q \neq 2$.

${ }^{6}$ Also here $q>2$, otherwise it is not scattered.
} 
Proposition 5.4. The linear set $\mathcal{L}$ is not PГL-equivalent to $L_{\delta}^{3}$.

Proof. By Lemma 5.2, we have to check whether $\mathcal{U}$ and $U_{\delta}^{3}$ are $\Gamma$ L-equivalent, with $\mathrm{N}_{q^{6} / q^{3}}(\delta) \notin\{0,1\}$. Suppose that there exist $\sigma \in \operatorname{Aut}\left(\mathbb{F}_{q^{6}}\right)$ and an invertible matrix $\left(\begin{array}{ll}a & b \\ c & d\end{array}\right)$ such that for each $x \in \mathbb{F}_{q^{6}}$ there exists $z \in \mathbb{F}_{q^{6}}$ satisfying

$$
\left(\begin{array}{ll}
a & b \\
c & d
\end{array}\right)\left(\begin{array}{c}
x^{\sigma} \\
x^{\sigma q}-x^{\sigma q^{2}}+x^{\sigma q^{4}}+x^{\sigma q^{5}}
\end{array}\right)=\left(\begin{array}{c}
z \\
\delta z^{q}+z^{q^{4}}
\end{array}\right) .
$$

Equivalently, for each $x \in \mathbb{F}_{q^{6}}$ we have

$$
\begin{gathered}
c x^{\sigma}+d\left(x^{\sigma q}-x^{\sigma q^{2}}+x^{\sigma q^{4}}+x^{\sigma q^{5}}\right)=\delta\left[a^{q} x^{\sigma q}+\right. \\
\left.+b^{q}\left(x^{\sigma q^{2}}-x^{\sigma q^{3}}+x^{\sigma q^{5}}+x^{\sigma}\right)\right]+a^{q^{4}} x^{\sigma q^{4}}+b^{q^{4}}\left(x^{\sigma q^{5}}-x^{\sigma}+x^{\sigma q^{2}}+x^{\sigma q^{3}}\right) .
\end{gathered}
$$

This is a polynomial identity in $x^{\sigma}$ and hence we have the following relations:

$$
\left\{\begin{array}{l}
c=\delta b^{q}-b^{q^{4}} \\
d=\delta a^{q} \\
-d=\delta b^{q}+b^{q^{4}} \\
0=-\delta b^{q}+b^{q^{4}} \\
d=a^{q^{4}} \\
d=\delta b^{q}+b^{q^{4}} .
\end{array}\right.
$$

From the second and the fifth equations, if $a \neq 0$ then $\delta=\left(a^{q}\right)^{q^{3}-1}$ and so $\mathrm{N}_{q^{6} / q^{3}}(\delta)=1$, which is not possible. So $a=0$ and then $d=0$. Hence we have $\delta b^{q}+b^{q^{4}}=0$ and $-\delta b^{q}+b^{q^{4}}=0$, from which we get $b=0$, which is not possible. Therefore, $\mathcal{L}$ is not equivalent to $L_{\delta}^{3}$.

Proposition 5.5. The linear set $\mathcal{L}$ is PCL-equivalent to $L_{\delta}^{4}$, for $q$ odd and $\delta^{2}+\delta=1$, if and only if there exist $a, b, c, d \in \mathbb{F}_{q^{6}}$ such that $a d-b c \neq 0$ and either

$$
\left\{\begin{array}{l}
c=b^{q}+\delta b^{q^{5}} \\
d=a^{q}+b^{q^{3}}-\delta b^{q^{5}} \\
-d=b^{q}+b^{q^{3}} \\
0=-b^{q}+a^{q^{3}}+\delta b^{q^{5}} \\
d=b^{q^{3}}+\delta b^{q^{5}} \\
d=b^{q}-b^{q^{3}}+\delta a^{q^{5}},
\end{array}\right.
$$


or

$$
\left\{\begin{array}{l}
c=\delta b^{q}+b^{q^{5}} \\
d=\delta a^{q}+b^{q^{3}}-b^{q^{5}} \\
-d=\delta b^{q}+b^{q^{3}} \\
0=-\delta b^{q}+a^{q^{3}}+b^{q^{5}} \\
d=b^{q^{3}}+b^{q^{5}} \\
d=\delta b^{q}-b^{q^{3}}+a^{q^{5}} .
\end{array}\right.
$$

In particular, when $q \equiv 0(\bmod 5)$ the linear set $\mathcal{L}$ is $\mathrm{P} \Gamma \mathrm{L}$-equivalent to $L_{2}^{4}$.

Proof. By Lemma 5.2 we have to check whether $\mathcal{U}$ is equivalent either to $U_{\delta}^{4}$ or to $\left(U_{\delta}^{4}\right)^{\perp}$. Suppose that there exist $\sigma \in \operatorname{Aut}\left(\mathbb{F}_{q^{6}}\right)$ and an invertible matrix $\left(\begin{array}{ll}a & b \\ c & d\end{array}\right)$ such that for each $x \in \mathbb{F}_{q^{6}}$ there exists $z \in \mathbb{F}_{q^{6}}$ satisfying

$$
\left(\begin{array}{ll}
a & b \\
c & d
\end{array}\right)\left(\begin{array}{c}
x^{\sigma} \\
x^{\sigma q}-x^{\sigma q^{2}}+x^{\sigma q^{4}}+x^{\sigma q^{5}}
\end{array}\right)=\left(\begin{array}{c}
z \\
z^{q}+z^{q^{3}}+\delta z^{q^{5}}
\end{array}\right) .
$$

Equivalently, for each $x \in \mathbb{F}_{q^{6}}$ we have

$$
\begin{gathered}
c x^{\sigma}+d\left(x^{\sigma q}-x^{\sigma q^{2}}+x^{\sigma q^{4}}+x^{\sigma q^{5}}\right)=a^{q} x^{\sigma q}+b^{q}\left(x^{\sigma q^{2}}-x^{\sigma q^{3}}+x^{\sigma q^{5}}+x^{\sigma}\right)+ \\
+a^{q^{3}} x^{\sigma q^{3}}+b^{q^{3}}\left(x^{\sigma q^{4}}-x^{\sigma q^{5}}+x^{\sigma q}+x^{\sigma q^{2}}\right)+ \\
+\delta\left[a^{q^{5}} x^{\sigma q^{5}}+b^{q^{5}}\left(x^{\sigma}-x^{\sigma q}+x^{\sigma q^{3}}+x^{\sigma q^{4}}\right)\right] .
\end{gathered}
$$

This is a polynomial identity in $x^{\sigma}$ and hence we have the Equations (9).

Now, suppose that there exist $\sigma \in \operatorname{Aut}\left(\mathbb{F}_{q^{6}}\right)$ and an invertible matrix $\left(\begin{array}{ll}a & b \\ c & d\end{array}\right)$ such that for each $x \in \mathbb{F}_{q^{6}}$ there exists $z \in \mathbb{F}_{q^{6}}$ satisfying

$$
\left(\begin{array}{ll}
a & b \\
c & d
\end{array}\right)\left(\begin{array}{c}
x^{\sigma} \\
x^{\sigma q}-x^{\sigma q^{2}}+x^{\sigma q^{4}}+x^{\sigma q^{5}}
\end{array}\right)=\left(\begin{array}{c}
z \\
\delta z^{q}+z^{q^{3}}+z^{q^{5}}
\end{array}\right) .
$$

As before, we get the Relations (10).

The second part follows from the fact that for $q \equiv 0(\bmod 5), \delta=2$, $a=-1, b=1, c=3$ and $d=3$ satisfy (9).

Thanks to GAP computations we are able to prove that the Systems (9) and (10) have no solutions in $a, b, c$ and $d(a c-b d \neq 0)$ for $q \leq 17$ and $q \not \equiv 0$ $(\bmod 5)$. Therefore, we have the following result. 
Corollary 5.6. If $q \leq 17, q \equiv 1(\bmod 4), q \neq 5$, then $\mathcal{L}$ is a maximum scattered linear set of $\mathrm{PG}\left(1, q^{6}\right)$ not equivalent to any of those listed in Example 5.1 .

Recall that $\mathcal{L}$ is computationally proved to be scattered for $q \leq 29, q \equiv 1$ $(\bmod 4)$.

We conclude this section proposing the following conjecture.

Conjecture 5.7. The linear set $\mathcal{L}$ is a new maximum scattered linear set of $\mathrm{PG}\left(1, q^{6}\right)$ for each $q$ such that $q \equiv 1(\bmod 4)$ and $q \not \equiv 0(\bmod 5)$.

\section{MRD-codes and scattered $\mathbb{F}_{q}$-subspaces}

The most natural way to look to the connection between maximum scattered linear sets and MRD-codes is through the $\mathbb{F}_{q}$-subspaces defining such linear sets, i.e. maximum scattered $\mathbb{F}_{q}$-subspaces. We briefly recall some basic definitions and results on rank metric codes, that have been intensively investigated for their applications in cryptography, space-time coding and distributed storage and for their links with remarkable geometric and algebraic objects (see e.g. [1, 18, 15, 30, 36, 39]).

In 1978, Delsarte [16] introduced rank metric codes as follows. The set of $m \times n$ matrices $\mathbb{F}_{q}^{m \times n}$ over $\mathbb{F}_{q}$ is a rank metric $\mathbb{F}_{q}$-space with rank metric distance defined by

$$
d(A, B)=\operatorname{rk}(A-B)
$$

for $A, B \in \mathbb{F}_{q}^{m \times n}$. A subset $\mathcal{C} \subseteq \mathbb{F}_{q}^{m \times n}$ is called a rank metric code (or $R M$ code for short). The minimum distance of $\mathcal{C}$ is

$$
d=\min \{d(A, B): A, B \in \mathcal{C}, A \neq B\} .
$$

We are interested in $\mathbb{F}_{q^{-}}$linear RM-codes, i.e. for which $\mathcal{C}$ is an $\mathbb{F}_{q}$-linear subspace of $\mathbb{F}_{q}^{m \times n}$. We will say that such a code has parameters $(m, n, q ; d)$. In [16], Delsarte also showed that the parameters of these codes must fulfill a Singleton-like bound, i.e.

$$
|\mathcal{C}| \leq q^{\max \{m, n\}(\min \{m, n\}-d+1)} .
$$

When the equality holds, we call $\mathcal{C}$ maximum rank distance ( $M R D$ for short) code. 
From now on, we will only consider $\mathbb{F}_{q}$-linear RM-codes of $\mathbb{F}_{q}^{n \times n}$, i.e. those which can be identified with $\mathbb{F}_{q^{-}}$-subspaces of $\operatorname{End}_{\mathbb{F}_{q}}\left(\mathbb{F}_{q^{n}}\right)$. Since $\operatorname{End}_{\mathbb{F}_{q}}\left(\mathbb{F}_{q^{n}}\right)$ is isomorphic to the ring of $q$-polynomials over $\mathbb{F}_{q^{n}}$ modulo $x^{q^{n}}-x$, denoted by $\mathcal{L}_{n, q}$, with addition and composition as operations, we will consider $\mathcal{C}$ as an $\mathbb{F}_{q}$-subspace of $\mathcal{L}_{n, q}$. Given two $\mathbb{F}_{q}$-linear RM-codes, $\mathcal{C}_{1}$ and $\mathcal{C}_{2}$, they are equivalent if and only if there exist $\varphi_{1}, \varphi_{2} \in \mathcal{L}_{n, q}$ permuting $\mathbb{F}_{q^{n}}$ and $\rho \in \operatorname{Aut}\left(\mathbb{F}_{q}\right)$ such that

$$
\varphi_{1} \circ f^{\rho} \circ \varphi_{2} \in \mathcal{C}_{2} \text { for all } f \in \mathcal{C}_{1},
$$

where $\circ$ stands for the composition of maps and $f^{\rho}(x)=\sum f_{i}^{\rho} x^{q^{i}}$ for $f(x)=$ $\sum f_{i} x^{q^{i}}$. For a rank metric code $\mathcal{C}$ given by a set of linearized polynomials, its left and right idealisers can be defined as:

$$
\begin{aligned}
& L(\mathcal{C})=\left\{\varphi \in \mathcal{L}_{n, q}: \varphi \circ f \in \mathcal{C} \text { for all } f \in \mathcal{C}\right\}, \\
& R(\mathcal{C})=\left\{\varphi \in \mathcal{L}_{n, q}: f \circ \varphi \in \mathcal{C} \text { for all } f \in \mathcal{C}\right\} .
\end{aligned}
$$

If $L(\mathcal{C})$ has maximum cardinality $q^{n}$, then we may always assume (up to equivalence) that

$$
L(\mathcal{C})=\mathcal{F}_{n}=\left\{\tau_{\alpha}=\alpha x: \alpha \in \mathbb{F}_{q^{n}}\right\} \simeq \mathbb{F}_{q^{n}} ;
$$

the same holds for the right idealiser, see [7, Theorem 6.1] and [8, Theorem 2.2]. Hence, when the left idealiser is $\mathcal{F}_{n}, \mathcal{C}$ results to be closed with respect to the left composition with the $\mathbb{F}_{q^{n}}$-linear maps; while if the right idealiser is $\mathcal{F}_{n}$, then $\mathcal{C}$ is closed with respect to the right composition with the $\mathbb{F}_{q^{n}}$-linear maps. For this reason, when $L(\mathcal{C})($ resp. $R(\mathcal{C}))$ is equal to $\mathcal{F}_{n}$ we say that $\mathcal{C}$ is $\mathbb{F}_{q^{n}}$-linear on the left (resp. right) (or simply $\mathbb{F}_{q^{n} \text {-linear if it is clear from }}$ the context).

The notion of Delsarte dual code can be written in terms of $q$-polynomials as follows, see for example [29, Section 2]. Let $b: \mathcal{L}_{n, q} \times \mathcal{L}_{n, q} \rightarrow \mathbb{F}_{q}$ be the bilinear form given by

$$
b(f, g)=\operatorname{Tr}_{q^{n} / q}\left(\sum_{i=0}^{n-1} f_{i} g_{i}\right)
$$

where $f(x)=\sum_{i=0}^{n-1} f_{i} x^{q^{i}}$ and $g(x)=\sum_{i=0}^{n-1} g_{i} x^{q^{i}}$, and $\operatorname{Tr}_{q^{n} / q}$ is the trace function $\mathbb{F}_{q^{n}} \rightarrow \mathbb{F}_{q}$. The Delsarte dual code $\mathcal{C}^{\perp}$ of a set of $q$-polynomials $\mathcal{C}$ is

$$
\mathcal{C}^{\perp}=\left\{f \in \mathcal{L}_{n, q}: b(f, g)=0, \forall g \in \mathcal{C}\right\} .
$$


Only few families of MRD-codes are known, due to the results in [1, 4, 33]. In [16], Delsarte gives the first construction for linear MRD-codes (he calls such sets Singleton systems) from the perspective of bilinear forms. Few years later, Gabidulin in [17, Section 4] presents the same class of MRD-codes by using linearized polynomials. Although these codes have been originally discovered by Delsarte, they are called Gabidulin codes. Kshevetskiy and Gabidulin in 21] generalize the previous construction obtaining the so-called generalized Gabidulin codes

$$
\mathcal{G}_{k, s}=\left\langle x, x^{q^{s}}, \ldots, x^{q^{s(k-1)}}\right\rangle_{\mathbb{F}_{q^{n}}},
$$

with $\operatorname{gcd}(s, n)=1$ and $k \leq n-1$. The RM-code $\mathcal{G}_{k, s}$ is an $\mathbb{F}_{q^{-}}$-linear MRDcode with parameters $(n, n, q ; n-k+1)$ and $L\left(\mathcal{G}_{k, s}\right)=R\left(\mathcal{G}_{k, s}\right) \simeq \mathbb{F}_{q^{n}}$, see $[22$, Lemma $4.1 \&$ Theorem 4.5]. Note that, as proved in [17, 21, this family is closed with respect to the Delsarte duality, more precisely $\mathcal{G}_{k, s}^{\perp}$ is equivalent to $\mathcal{G}_{n-k, s}$. This family of MRD-codes has been characterized by HorlemannTrautmann and Marshall in [20] as follows.

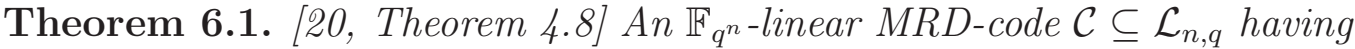
dimension $k$ (over $\mathbb{F}_{q^{n}}$ ) is equivalent to a generalized Gabidulin code $\mathcal{G}_{k, s}$ if and only if there is an integer $s<n$ with $\operatorname{gcd}(s, n)=1$ and $\operatorname{dim}_{\mathbb{F}_{q^{n}}}\left(\mathcal{C} \cap \mathcal{C}^{[s]}\right)=$ $k-1$, where $\mathcal{C}^{[s]}=\left\{f(x)^{q^{s}}: f \in \mathcal{C}\right\}$.

Very recently, Neri in 32] removed the hypothesis on $\mathcal{C}$ to be an MRDcode.

Sheekey in [37] proves that with $\operatorname{gcd}(s, n)=1$, the set

$$
\mathcal{H}_{k, s}(\eta, h)=\left\{a_{0} x+a_{1} x^{q^{s}}+\ldots+a_{k-1} x^{q^{s(k-1)}}+a_{0}^{q^{h}} \eta x^{q^{s k}}: a_{i} \in \mathbb{F}_{q^{n}}\right\},
$$

with $k \leq n-1$ and $\eta \in \mathbb{F}_{q^{n}}$ such that $\mathrm{N}_{q^{n} / q}(\eta) \neq(-1)^{n k}$, is an $\mathbb{F}_{q^{-}}$-linear MRD-code of dimension $n k$ with parameters $(n, n, q ; n-k+1)$. This code is called generalized twisted Gabidulin code. Lunardon, Trombetti and Zhou in [29], generalizing the results of [37], determined the automorphism group of the generalized twisted Gabidulin codes and proved that, up to equivalence, the generalized Gabidulin codes and the twisted Gabidulin codes are both proper subsets of this class. Clearly, for $\eta=0$ we have exactly the generalized Gabidulin code $\mathcal{G}_{k, s}$. Also, the authors in [29, Corollary 5.2] determined the left and right idealisers: if $\eta \neq 0$, then

$$
L\left(\mathcal{H}_{k, s}(\eta, h)\right) \simeq \mathbb{F}_{q^{\operatorname{gcd}(n, h)}} \text { and } R\left(\mathcal{H}_{k, s}(\eta, h)\right) \simeq \mathbb{F}_{q^{\operatorname{gcd}(n, s k-h)}}
$$


The class of generalized twisted Gabidulin codes is closed with respect to the Delsarte duality, more precisely $\mathcal{H}_{k, s}(\eta, h)^{\perp}$ is equivalent to $\mathcal{H}_{n-k, s}(-\eta, n-h)$, [37, Theorem 6] and [29, Propositions 4.2]. We are interested in the case when $h=0$, i.e.

$$
\mathcal{H}_{k, s}(\eta):=\mathcal{H}_{k, s}(\eta, 0)=\left\langle x+\eta x^{q^{s k}}, x^{q^{s}}, \ldots, x^{q^{s(k-1)}}\right\rangle_{\mathbb{F}_{q^{n}}}
$$

which is $\mathbb{F}_{q^{n}}$-linear (more precisely it is an $\mathbb{F}_{q^{-}}$-linear MRD-code $\mathbb{F}_{q^{n}}$-linear on the left). This family has been characterized in [19].

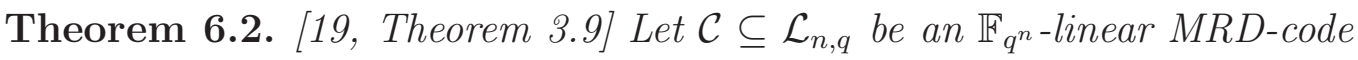
having dimension $k>2$. Then, the code $\mathcal{C}$ is equivalent to a generalized twisted Gabidulin code if and only if there exists an integer s such that $\operatorname{gcd}(s, n)=1$ and such that the following two conditions hold

1. $\operatorname{dim}\left(\mathcal{C} \cap \mathcal{C}^{[s]}\right)=k-2$ and $\operatorname{dim}\left(\mathcal{C} \cap \mathcal{C}^{[s]} \cap \mathcal{C}^{[2 s]}\right)=k-3$, i.e. there exist $p(x), q(x) \in \mathcal{C}$ such that

$$
\mathcal{C}=\left\langle p(x)^{q^{s}}, p(x)^{q^{2 s}}, \ldots, p(x)^{q^{s(k-1)}}\right\rangle_{\mathbb{F}_{q^{n}}} \oplus\langle q(x)\rangle_{\mathbb{F}_{q^{n}}}
$$

2. $p(x)$ is invertible and there exists $\eta \in \mathbb{F}_{q^{n}}^{*}$ such that $p(x)+\eta p(x)^{q^{s k}} \in \mathcal{C}$.

Apart from the two infinite families of $\mathbb{F}_{q^{n}}$-linear MRD-codes (i.e. $\mathcal{G}_{k, s}$ and $\left.\mathcal{H}_{k, s}(\eta)\right)$, there are few other examples known for $n \in\{6,7,8\}$, which arise from the connection with scattered linear sets we are going to explain.


of dimension $n$ yield $\mathbb{F}_{q}$-linear MRD-codes with parameters $(n, n, q ; n-1)$ with left idealiser isomorphic to $\mathbb{F}_{q^{n}}$; see [9, 10, 38] for further details on such kind of connections. Let us recall the construction from [37]. Let

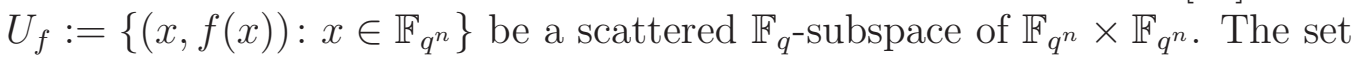

$$
\mathcal{C}_{f}:=\langle x, f(x)\rangle_{\mathbb{F}_{q^{n}}}
$$

corresponds to a set of $n \times n$ matrices over $\mathbb{F}_{q}$ forming an $\mathbb{F}_{q}$-linear MRDcode with parameters $(n, n, q ; n-1)$. Also, since $\mathcal{C}_{f}$ is an $\mathbb{F}_{q^{n} \text {-subspace of }}$ $\mathcal{L}_{n, q}$, its left idealiser $L\left(\mathcal{C}_{f}\right)$ is isomorphic to $\mathbb{F}_{q^{n}}$. For further details see [7. Section 6]. Furthermore, let $\mathcal{C}_{f}$ and $\mathcal{C}_{h}$ be two MRD-codes arising from maximum scattered subspaces $U_{f}$ and $U_{h}$ of $\mathbb{F}_{q^{n}} \times \mathbb{F}_{q^{n}}$. In [37, Theorem 8] the author showed that there exist invertible matrices $A, B$ and $\sigma \in \operatorname{Aut}\left(\mathbb{F}_{q}\right)$ 
such that $A \mathcal{C}_{f}^{\sigma} B=\mathcal{C}_{h}$ if and only if $U_{f}$ and $U_{h}$ are $\Gamma \mathrm{L}\left(2, q^{n}\right)$-equivalent, i.e. he proved that the equivalence of the rank metric codes coincides with the TL-equivalence of the corresponding subspaces.

As a consequence we get the following result.

Theorem 6.3. If $q \leq 17, q \equiv 1(\bmod 4)$ and $q \neq 5$, then the $R M$-code $\mathcal{C}=\left\langle x, x^{q}-x^{q^{2}}+x^{q^{4}}+x^{q^{5}}\right\rangle_{\mathbb{F}_{q^{6}}}$ is an $\mathbb{F}_{q^{-}}$-linear MRD-code with parameters $(6,6, q ; 5)$ and left idealiser isomorphic to $\mathbb{F}_{q^{6}}$, and is not equivalent to any previously known MRD-code.

Proof. From [7, Section 6], the previously known $\mathbb{F}_{q^{-}}$linear MRD-codes with parameters $(6,6, q ; 5)$ and with left idealiser isomorphic to $\mathbb{F}_{q^{6}}$ arise, up to equivalence, from one of the maximum scattered subspaces of $\mathbb{F}_{q^{6}} \times \mathbb{F}_{q^{6}}$ described in Section 5.1. From Corollary 5.6 the result then follows.

\subsection{Scattered linear sets and MRD-codes}

Lunardon in [24, Section 3] (see also [38, Theorem 3.4] and [10, Section 4.1]) proved that if $U_{f}=\left\{(x, f(x)): x \in \mathbb{F}_{q^{n}}\right\}$, with $f(x)=a_{0} x+a_{1} x^{q}+\ldots+$ $a_{n-1} x^{q^{n-1}}$, is a scattered $\mathbb{F}_{q^{-}}$-subspace of $\mathbb{F}_{q^{n}} \times \mathbb{F}_{q^{n}}$, then it can be obtained as a special quotient. By [37, Section 5], it follows that

$$
\mathcal{C}_{f}=\langle x, f(x)\rangle_{\mathbb{F}_{q^{n}}}
$$

is an MRD-code. We may assume that the coefficient of $x$ in $f(x)$ is zero and $f(x)=x^{q^{k}}+\sum_{j \neq k} b_{j} x^{q^{j}}$. Denoting with $\left\{i_{1}, \ldots, i_{n-2}\right\}=\{1, \ldots, k-1, k+$ $1, \ldots, n-1\}$ and

$$
h_{i_{j}}(x)=x^{q^{i_{j}}}-b_{i_{j}} x^{q^{k}}, \quad j=1, \ldots, n-2,
$$

it is straightforward to see that

$$
\mathcal{C}^{\perp}=\left\langle h_{i_{1}}(x), \ldots, h_{i_{n-2}}(x)\right\rangle_{\mathbb{F}_{q^{n}}} .
$$

We can embed $U_{f}$ in $\mathbb{F}_{q^{n}}^{n}$ in such a way that the vector $(x, f(x))$ corresponds to the vector $\left(a_{0}, \ldots, a_{n-1}\right) \in \mathbb{F}_{q^{n}}^{n}$ with $a_{i}=0$ if $i \neq 0, k, a_{0}=x$ and $a_{k}=f(x)$. Note that $W=\left\langle U_{f}\right\rangle_{\mathbb{F}_{q^{n}}}$ corresponds to the 2-dimensional subspace with equations $x_{j}=0$ where $j \neq 0, k$.

\footnotetext{
${ }^{7}$ The statement is more general, we have adapted it to our case.
} 



equations

$$
V:\left\{\begin{array}{l}
x_{0}=0 \\
x_{k}=-\sum_{j \neq 0, k} b_{j} x_{j}
\end{array},\right.
$$

and let $S=\left\{\left(x, x^{q}, \ldots, x^{q^{n-1}}\right): x \in \mathbb{F}_{q^{n}}\right\}$. Note that

$$
V=c_{\mathcal{N}}\left(\mathcal{C}^{\perp}\right)
$$

where $c_{\mathcal{N}}\left(\alpha_{0} x+\ldots+\alpha_{n-1} x^{q^{n-1}}\right)=\left(\alpha_{0}, \ldots, \alpha_{n-1}\right)$. It can be seen that $V \cap S=$ $\{\mathbf{0}\}$ and

$$
U_{f}=\langle V, S\rangle_{\mathbb{F}_{q}} \cap W
$$

This link suggests a new proof of the equivalence between the assertions 1. and 2. of Theorem 1.1. In the following we will assume that $L=L_{f}$ is a scattered linear set of $\mathrm{PG}\left(1, q^{n}\right)$ with rank $n$.

Proof. (Theorem 1.1) Assume that $L_{f}$ is of pseudoregulus type, then by [12] we have that if $L_{U}=L_{f}$ then $U$ is $\Gamma \mathrm{L}\left(2, q^{n}\right)$-equivalent to

$$
U_{s}=\left\{\left(x, x^{q^{s}}\right): x \in \mathbb{F}_{q^{n}}\right\} \text {. with } \operatorname{gcd}(s, n)=1 \text { and } s<n / 2 .
$$

Therefore if $U=U_{s}$, then $U_{s}=\langle V, S\rangle_{\mathbb{F}_{q}} \cap W$, with

$$
V:\left\{\begin{array}{l}
x_{0}=0 \\
x_{s}=0
\end{array} \text { and } W: x_{i}=0 \text { for } i \neq 0, s,\right.
$$

i.e. $L_{f}=p_{\Gamma, \Lambda}(\Sigma)$ with $\Gamma=\operatorname{PG}\left(V, \mathbb{F}_{q^{n}}\right)=\operatorname{PG}\left(n-3, q^{n}\right), \Sigma=\mathrm{PG}\left(S, \mathbb{F}_{q}\right)=$ $\mathrm{PG}(n-1, q)$ and $\Lambda=\mathrm{PG}\left(W, \mathbb{F}_{q^{n}}\right)=\mathrm{PG}\left(1, q^{n}\right)$. Denote by $\sigma$ the collineation of $\operatorname{PG}\left(n-1, q^{n}\right)$ defined by $\left\langle\left(x_{0}, \ldots, x_{n-1}\right)\right\rangle_{\mathbb{F}_{q^{n}}}^{\sigma}=\left\langle\left(x_{n-1}^{q}, x_{0}^{q}, \ldots, x_{n-2}^{q}\right)\right\rangle_{\mathbb{F}_{q^{n}}}$, which fixes precisely the points of $\Sigma$. Therefore, we have that $\operatorname{dim}\left(\Gamma \cap \Gamma^{\sigma^{s}}\right)=$ $n-4$ and clearly $\sigma^{s}$ is a generator of the subgroup of $\operatorname{P\Gamma L}\left(n, q^{n}\right)$ fixing $\Sigma$ pointwise.

Conversely, let $L=p_{\Gamma, \Lambda}(\Sigma)$ with $\operatorname{dim}\left(\Gamma \cap \Gamma^{\sigma^{s}}\right)=n-4, \operatorname{gcd}(s, n)=1$, $\Gamma=\operatorname{PG}\left(V, \mathbb{F}_{q^{n}}\right)=\operatorname{PG}\left(n-3, q^{n}\right), \Sigma=\operatorname{PG}\left(S, \mathbb{F}_{q}\right)=\operatorname{PG}(n-1, q)$. Note that $V=c_{\mathcal{N}}(\mathcal{C})$ with

$$
\mathcal{C}=\left\langle g_{1}(x), \ldots, g_{n-2}(x)\right\rangle_{\mathbb{F}_{q^{n}}},
$$

for some linearized polynomials $g_{1}(x), \ldots, g_{n-2}(x)$. It follows that

$$
V:\left\{\begin{array}{l}
a_{0} x_{0}+\ldots+a_{n-1} x_{n-1}=0 \\
a_{0}^{\prime} x_{0}+\ldots+a_{n-1}^{\prime} x_{n-1}=0
\end{array},\right.
$$


where $\mathcal{C}^{\perp}=\left\langle f_{1}(x), f_{2}(x)\right\rangle_{\mathbb{F}_{q^{n}}}$ and

$$
\begin{aligned}
& f_{1}(x)=a_{0} x+\ldots+a_{n-1} x^{q^{n-1}} \\
& f_{2}(x)=a_{0}^{\prime} x+\ldots+a_{n-1}^{\prime} x^{q^{n-1}} .
\end{aligned}
$$

We may assume that $a_{j}=a_{k}^{\prime}=1$ and $a_{k}=a_{j}^{\prime}=0$ for some $j \neq k$, choose $W$

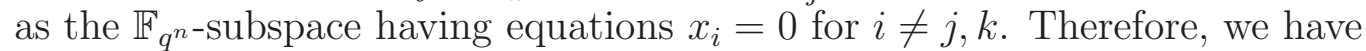

$$
(V+S) \cap W \simeq U:=\left\{\left(f_{1}(x), f_{2}(x)\right): x \in \mathbb{F}_{q^{n}}\right\}
$$

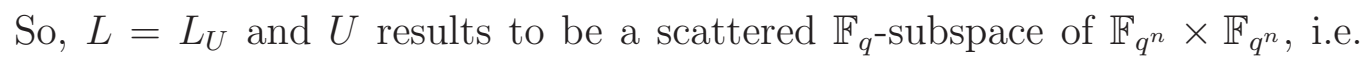
by [37, Section 5] $\mathcal{C}^{\perp}$ is an MRD-code. It follows that $\mathcal{C}$ is an MRD-code with $\operatorname{dim}_{\mathbb{F}_{q^{n}}} \mathcal{C}=n-2$ and $\operatorname{dim}_{\mathbb{F}_{q^{n}}}\left(\mathcal{C} \cap \mathcal{C}^{[s]}\right)=n-3$. By Theorem 6.1, $\mathcal{C}$ is equivalent to $\mathcal{G}_{n-2, s}$. It follows that $U$ is $\Gamma \mathrm{L}\left(2, q^{n}\right)$-equivalent to $U_{s}$ and hence $L$ is of pseudoregulus type.

In [32, Neri gives a characterization of generalized Gabidulin codes using the standard form of their generator matrix. This suggests a further different approach to the characterization of linear sets of pseudoregulus type.

For linear sets of LP-type, as done for the pseudoregulus case, it follows that one of the possible $\mathbb{F}_{q}$-subspaces representing a linear set of LP-type can be obtained as in (13), choosing $V$ in such a way that $V=c_{\mathcal{N}}\left(\mathcal{H}_{n-2, s}(\eta)\right)$. Since a characterization of generalized twisted Gabidulin codes is known, see Theorem 6.2 with $k=n-2$, it follows that a scattered linear set $L$ is of

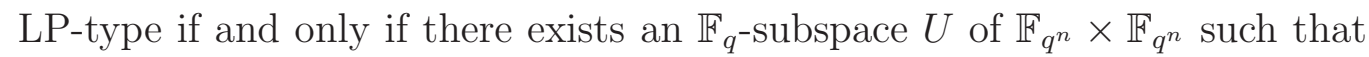
$L_{U}=L$, where $U$ is as in (13) and the rank-metric code associated to $V$ satisfies the hypothesis of Theorem 6.2 with $k=n-2$. In contrast to the above characterization, those presented in the previous sections are purely geometric and take into account the problem of the possible $\mathbb{F}_{q^{-} \text {-subspaces }}$ representing a linear set of LP-type.

\section{References}

[1] D. Bartoli and Y. Zhou: Exceptional scattered polynomials, J. Algebra 509 (2018), 507-534.

[2] A. Blokhuis and M. Lavrauw: Scattered spaces with respect to a spread in PG(n,q), Geom. Dedicata 81 (2000), 231-243. 
[3] G. Bonoli and O. Polverino: $\mathbb{F}_{q}$-linear blocking sets in $\operatorname{PG}\left(2, q^{4}\right)$, Innov. Incidence Geom. 2 (2005): 35-56.

[4] sc Byrne E., Ravagnani, A.: Partition-balanced families of codes and asymptotic enumeration in coding theory, https://arxiv.org/abs/1805.02049.

[5] B. Csajbók, G. Marino and O. Polverino: Classes and equivalence of linear sets in PG(1, $\left.q^{n}\right)$, J. Combin. Theory Ser. A 157 (2018), 402-426.

[6] B. Csajbók, G. Marino and O. Polverino: A Carlitz type result for linearized polynomials, Ars Math. Contemp. 16(2)(2019), 585-608.

[7] B. Csajbók, G. Marino, O. Polverino and C. Zanella: A new family of MRD-codes, Linear Algebra Appl. 548 (2018), 203-220.

[8] B. Csajbók, G. Marino, O. Polverino and Y. Zhou: Maximum Rank-Distance codes with maximum left and right idealisers, https://arxiv.org/abs/1807.08774.

[9] B. Csajbók, G. Marino, O. Polverino and F. Zullo: Maximum scattered linear sets and MRD-codes, J. Algebraic Combin. 46 (2017), 1-15.

[10] B. Csajbók, G. Marino, O. Polverino and F. Zullo: A special class of scattered subspaces, arXiv:1906.10590.

[11] B. Csajbók, G. Marino and F. Zullo: New maximum scattered linear sets of the projective line, Finite Fields Appl. 54 (2018), 133-150.

[12] B. Csajbók and C. Zanella: On the equivalence of linear sets, Des. Codes Cryptogr. 81 (2016), 269-281.

[13] B. Csajbók and C. Zanella: On scattered linear sets of pseudoregulus type in PG(1, $\left.q^{t}\right)$, Finite Fields Appl. 41 (2016), 34-54.

[14] B. Csajbók And C. Zanella: Maximum scattered $\mathbb{F}_{q}$-linear sets of PG $\left(1, q^{4}\right)$, Discrete Math. 341 (2018), 74-80. 
[15] J. de la Cruz, M. Kiermaier, A. Wassermann and W. Willems: Algebraic structures of MRD Codes, Adv. Math. Commun. 10 (2016), 499-510.

[16] P. Delsarte: Bilinear forms over a finite field, with applications to coding theory, J. Combin. Theory Ser. A 25 (1978), 226-241.

[17] E. Gabidulin: Theory of codes with maximum rank distance, Problems of information transmission, 21(3) (1985), 3-16.

[18] E. M. Gabidulin, A. Paramonov and O. Tretuakov: Ideals over a Non-Commutative Ring and Their Application in Cryptology, in Workshop on the Theory and Application of of Cryptographic Techniques, Springer (1991), 482-489.

[19] L. Giuzzi AND F. Zullo: Identifiers for MRD-codes, Linear Algebra Appl. 575 (2019), 66-86.

[20] A. Horlemann-Trautmann and K. Marshall: New criteria for MRD and Gabidulin codes and some rank-metric code constructions, Adv. Math. Commun. 11(3) (2017), 533-548.

[21] A. Kshevetskiy and E. Gabidulin: The new construction of rank codes, International Symposium on Information Theory, 2005. ISIT 2005. Proceedings, pages 2105-2108, Sept. 2005.

[22] D. Liebhold and G. NeBe: Automorphism groups of Gabidulin-like codes, Arch. Math. 107(4) (2016), 355-366.

[23] G. Lunardon: Normal spreads, Geom. Dedicata 75 (1999), 245-261.

[24] G. Lunardon: MRD-codes and linear sets, J. Combin. Theory Ser. A 149 (2017), 1-20.

[25] G. Lunardon, G. Marino, O. Polverino and R. Trombetti: Maximum scattered linear sets of pseudoregulus type and the Segre Variety $\mathcal{S}_{n, n}$, J. Algebraic. Combin. 39 (2014), 807-831.

[26] G. Lunardon, P. Polito and O. Polverino: A geometric characterisation of linear k-blocking sets, J. Geom. 74 (1-2) (2002), 120-122. 
[27] G. Lunardon and O. Polverino: Blocking Sets and Derivable Partial Spreads, J. Algebraic Combin. 14 (2001), 49-56.

[28] G. Lunardon and O. Polverino: Translation ovoids of orthogonal polar spaces, Forum Math. 16 (2004), 663-669.

[29] G. Lunardon, R. Trombetti and Y. Zhou: Generalized Twisted Gabidulin Codes, J. Combin. Theory Ser. A 159 (2018), 79-106.

[30] P. Lusina, E. M. Gabidulin and M. Bossert: Maximum rank distance codes as space-time codes, IEEE Trans. Info. Theory 49 (2003), $2757-2760$.

[31] G. Marino, M. Montanucci and F. Zullo: MRD-codes arising from the trinomial $x^{q}+x^{q^{3}}+c x^{q^{5}} \in \mathbb{F}_{q^{6}}[x]$, arXiv:1907.08122.

[32] A. NeRI: Systematic encoders for generalized Gabidulin codes and the $q$-analogue of Cauchy matrices, https://arxiv.org/abs/1805.06706.

[33] A. Neri, A. Horlemann-Trautmann, T. Randrianarisoa and J. Rosenthal: On the genericity of maximum rank distance and Gabidulin codes, Des. Codes Cryptogr. 86(2) (2018), 1-23.

[34] A. Neri, S. Puchinger, A. Horlemann-Trautmann: Invariants and Inequivalence of Linear Rank-Metric Codes, arXiv:1905.11326.

[35] A. Neri, S. Puchinger, A. Horlemann-Trautmann: Equivalence and Characterizations of Linear Rank-Metric Codes Based on Invariants, arXiv:1911.13059.

[36] A. Neri, J. Rosenthal and D. Schipani: Fuzzy Authentication using Rank Distance, arXiv:1703.03235.

[37] J. Sheekey: A new family of linear maximum rank distance codes, Adv. Math. Commun. 10(3) (2016), 475-488.

[38] J. Sheekey and G. Van de Voorde: Rank-metric codes, linear sets and their duality, arXiv:1806.05929.

[39] N. Silberstein, A.S. Rawat, O.O. Koyluoglu and S. VishWANATH: Optimal locally repairable codes via rank-metric codes, IEEE Int. Symp. Inf. Theory (ISIT) (2013), Istanbul (Turkey). 
[40] F. Zullo: Linear codes and Galois geometries: between two worlds, Ph.D. thesis, Università degli Studi della Campania "Luigi Vanvitelli".

Corrado Zanella and Ferdinando Zullo

Dipartimento di Tecnica e Gestione dei Sistemi Industriali

Università degli Studi di Padova

Stradella S. Nicola, 3

36100 Vicenza VI

Italy

\{corrado.zanella,ferdinando.zullo\}@unipd.it

Ferdinando Zullo

Dipartimento di Matematica e Fisica,

Università degli Studi della Campania "Luigi Vanvitelli",

I-81100 Caserta, Italy

ferdinando.zullo@unicampania.it 